\title{
A laser-cooled atomic probe for low variations of static magnetic fields.
}

\author{
S. Ghezali and A. Clairon(*) \\ Department of physics. Faculty of sciences. University of Blida. Blida 09000. Algeria. \\ (*): SYRTE. Paris Observatory. 77, Avenue Denfert-Rochereau. Paris 75014. France.
}

\begin{abstract}
Neutral atom trapping and laser cooling has opened the way to large prospects for the present generation of atomic frequency standards [1]. A cold cesium fountain device has been built for the first time at the Observatoire de Paris and is now operating since 1994. Its frequency stability, $410^{-14} \tau^{-1 / 2}$ where $\tau$ is the integration time, has been the best one ever measured for a while. This letter shows how accurate can be the measurement of low static magnetic fields within the fountain device and the variations along the longitudinal and transverse axis. Thus the device is no more operating as a clock but constitutes a very precise magnetometer.
\end{abstract}

\section{Introduction}

An atomic frequency standard based on a fountain of cold cesium atoms has been in operation at the Observatoire de Paris since about a decade. It has been included in the calculation of the international atomic time because of its great performances. The flight time between both $\pi / 2$ Ramsey interactions is lengthened to about $500 \mathrm{~ms}$ so that Ramsey fringes as narrow as $700 \mathrm{mHz}$ have been measured [1] for cesium atoms of few $\mu \mathrm{K}$ using high power laser diodes at the infrared wavelength $852 \mathrm{~nm}$. This frequency standard is three to four orders of magnitude better than classical thermal beam devices in frequency stability and accuracy.

\section{The prototype}

This fountain device has been fully described in many papers [1] and a $\mathrm{PhD}$ manuscripts [2,3]. It is constituted by three main zones: the trapping and cooling zone, the microwave and the C-field zone and the optical detection zone (figure 1). The atoms are first trapped and pre-cooled in a magneto-optical trap or an optical molasses for $1 \mathrm{~s}$ or so, accelerated through a 1D or 3D moving molasses technique and then cooled again by sub-Doppler cooling for just few ms. After launching, the atoms are in $\mathrm{F}=4(\mathrm{~F}=3)$, one of the two hyperfine states of the ground level $6 \mathrm{~S}_{1 / 2}$, pass through a cylindrical Ramsey cavity of a high quality factor where they experience a first $\pi / 2$ pulse, continue their ballistic flight in the darkness in the C-field region where a highly homogeneous static magnetic field is produced by a solenoid and compensation coils, pass a second time through the microwave cavity where they undergo a second $\pi / 2$ pulse and are transferred to the second hyperfine state $\mathrm{F}=3(\mathrm{~F}=4)$ and then are detected with two laser probe beams. The atomic populations are measured via a time of flight technique through fluorescence which is analysed in real time and digitized by a personal computer. The normalisation procedure reduces the fluctuations of the detection signal from shot to shot which are typically $1 \%$.

\section{Results}

Figure 2 shows Ramsey fringes and a central resonance $700 \mathrm{mHz}$ wide, two orders of magnitude narrower than in the best thermal beam device. The microwave interrogation signal at $9.19263177 \mathrm{GHz}$ switches from one side of the resonance to the other for successive fountain cycles. The difference between both transition probabilities is calculated giving an error signal that is used to control numerically the frequency of the synthesizer of the frequency chain used to generate the $9.2 \mathrm{GHz}$ [2]. This allows frequency stability measurements of the fountain against the Hydrogen maser $(100 \mathrm{MHz})$ to which is weakly phase-locked a $10 \mathrm{MHz}$ quartz oscillator.The expected fractionnal frequency stability can be evaluated by the square root of the two-sample Allan variance:

$$
\sigma(\tau)=\frac{\delta v}{v_{0}} \frac{N}{S}\left\{\sqrt{\frac{T_{C}}{\tau}}\right\}^{-1 / 2}
$$


where $v_{0}$ is the clock frequency, $\delta v$ is the width of the central resonance, $\mathrm{S} / \mathrm{N}$ the signal to noise ratio noted $\mathrm{SNR}, \mathrm{T}_{\mathrm{C}}$ is the duration of one complete cycle and $\tau$ is the integration time. Typical parameters correspond to a pure optical molasses loading, ie $10^{5}$ atoms in $\mathrm{M}_{\mathrm{F}}=0$ at the detection, a signal to noise ratio of 1000 for each launching and a $1 \mathrm{~Hz}$ central fringe. Initially, the measured frequency stability shown in figure 3 was $1.310^{-13} \tau^{-}$ $1 / 2$ which is less than the expected one (given by equation 1). It has been improved to $410^{-14} \tau^{-1 / 2}$ some time later. For $10^{4} \mathrm{~s}$ of integration time, the measured frequency stability reaches that of the Hydrogen maser, ie 1 $10^{-15}$. The observed degradation of the stability is due to an aliasing effect where the local oscillator high frequency noise is down converted to low frequency inducing the degradation of the signal to noise at the detection. For such a reason, a cryogenic saphir oscillator from the University of Western Australia has been used and a frequency stability as good as $410^{-14} \tau^{-1 / 2}$ has been measured [3]. This experiment has proved that the short term frequency stability is only limited by the atomic projection noise. As pointed out in [4], this is a binomial statistical atomic noise which is maximum at half the linewidth of the central Ramsey fringe.

All the frequency shifts measurements are limited by the resolution of the fountain device given by the frequency instabilities of the Hydrogen maser which have been improved to $710^{-16}$ with the help of external compensation coils around the Hydrogen maser commercial setup.

\section{A magnetic probe:}

In the fountain, the two Ramsey interactions occur in the same and unique microwave cavity so that all the phase effects within the cavity are reduced. Atoms can be loaded in a magneto-optical trap in order to increase their sensitivity to the transverse phase gradient of the field's distribution. At the first passage, the atomic cloud's size is only $2 \mathrm{~mm}$ compared to the $5 \mathrm{~mm}$ of radius of the hole of the cavity. The microwave field varies of about $20 \%$ between the center and the edge of the hole of the cavity. This induces an attenuation of the Rabi oscillations (figure 4).Mapping the static magnetic field in a cold atomic fountain can be useful for many purposes such as the Zeeman quadratic frequency shift and as well as the evaluation of the collisional frequency shifts [5]. In thermal beam frequency standards, the static magnetic field is calculated from the frequency difference of two adjacent microwave lines $\Delta M_{F}=0$, the quadratic frequency dependant magnetic field (clock transition) $\mathrm{F}=4, \mathrm{M}_{\mathrm{F}}=0 \rightarrow \mathrm{F}=3, \mathrm{M}_{\mathrm{F}}=0$ and a linearly frequency dependant magnetic field for instance $\mathrm{F}=4, \mathrm{M}_{\mathrm{F}}=+1 \rightarrow \mathrm{F}=4$,

$\mathrm{M}_{\mathrm{F}}=+1$ transition (see figure 5). This is the mean value seen by the atoms during the free flight between the two $\frac{\pi}{2}$ microwave Ramsey interactions.

In a cold atomic fountain, this can be undergone by launching atoms to different heights above the Ramsey cavity. As long as the central Ramsey fringe of both the clock transition and the nearest $\sigma$ transition (see figure 6) is easily identified through the maximum contrast criterion, the mean longitudinal magnetic field is calculated in that way [3].

When the atoms'launching overcomes typically $10 \mathrm{~cm}$ above the Ramsey cavity, the fringes'number becomes so large that it is hard to identify the central Ramsey fringe. In this case, we apply a $\pi$ microwave pulse at the atoms'apogee through an antenna placed at the top of the fountain device (see figure 1). This cancels the first Doppler frequency shift and the frequency difference between the two first $\sigma$ transitions

$\mathrm{M}_{\mathrm{F}}=+1 \rightarrow \mathrm{M}_{\mathrm{F}}=+1$ and $\mathrm{M}_{\mathrm{F}}=-1 \rightarrow \mathrm{M}_{\mathrm{F}}=-1$ gives access to the local magnetic field with a resolution of $0.1 \mu G$. The atoms don't move during these $10 \mathrm{~ms}$ of duration of the applied $\pi$ microwave pulse. Because of the huge difference between the magnetic field inside the Ramsey cavity denoted $\mathrm{B}_{\text {cav }}$ and the mean magnetic field $\langle\mathrm{B}\rangle$ above the Ramsey cavity, the fringes are so displaced that the central fringe is within the wing of the Rabi envelope.

Combining these two measurement techniques, a map of the longitudinal magnetic field can be done. Figure 7 shows the axial magnetic field map before any correction. The zero altitude corresponds to a height of $29 \mathrm{~cm}$ above the cooling zone. The mean value of the magnetic field is not the same for all the atomic trajectories because of magnetic inhomogeneities. To compute a linearly dependant magnetic field transition, one has to average $<\mathrm{B}>$ over all the possible atomic ways. Taking into account the cloud's spatial extension, the transverse and axial magnetic inhomogeneities are estimated to the level of $1 \mu G / \mathrm{cm}^{3}$. The calculation of $\mathrm{B}(\mathrm{z})$ allows one to deduce the quantity $\left\langle\mathrm{B}^{2}(\mathrm{t})\right\rangle$ used in the determination of the second order Zeeman frequency shift. In order to improve the continuity of the magnetic field, we use the compensation coils placed between the $\mu$ metal magnetic shields at at both their extremities. Figure 8 shows the $F=4, M_{F}=-1 \rightarrow F=3, M_{F}=-1$ transition after correction. 
We see clearly that the difference between the magnetic field in the cavity and outside the cavity is much smaller after correction:

$$
<\mathrm{B}>-\mathrm{B}_{\mathrm{cav}}=4 \mu G
$$

On the other hand, modulating the Ramsey fringes allows to reduce the number into the Rabi enveloppe so that the central fringe is easily and precisely identified (see figure 9).

One has simply to detune slightly the horizontal laser beams by a quantity $\Delta v$ of few tens of $\mathrm{kHz}$ from resonance. The result is the creation of three longitudinal velocity distributions and thus three pics in the time of flight signal separated by $\Delta v=\Delta v . \lambda$ where $\lambda$ is the infrared transition wavelength (see figure 10). We can improve the level of uncertainty on the central fringe determination by measuring the frequency difference between $\mathrm{F}=4, \mathrm{M}_{\mathrm{F}}=0 \rightarrow \mathrm{F}=3, \mathrm{M}_{\mathrm{F}}=0$ and $\mathrm{F}=4, \mathrm{M}_{\mathrm{F}}=2 \rightarrow \mathrm{F}=3, \mathrm{M}_{\mathrm{F}}=2$. The relative uncertainty in this case is about $10^{-16}$. We have:

$$
\begin{array}{r}
\mathrm{B}(\mathrm{t})=\langle\mathrm{B}>+\varepsilon(\mathrm{t}) \\
\left\langle B^{2}>-<B>^{2}=\int_{T_{V}}^{0} \frac{1}{T_{V}} \varepsilon^{2}(t) d t\right.
\end{array}
$$

where $\mathrm{T}_{\mathrm{V}}$ is the duration of the free flight above the Ramsey cavity. Admitting that $\varepsilon(\mathrm{t})$ is about 10 $\mu G$, the relative uncertainty on the magnetic field due to this term is about $5.10^{-18}$. This value allows to consider the cold fountain device as an ideal but expensive magnetometer [3]. The short term stabilty of the static magnetic field is given by figure 11 . We have no explanation for this kind of variation(sinusoidal) over time. Also the atoms don't pass by the center of the microwave cavity so that they will see a microwave field which is not parallel to the static magnetic field. This type of interaction induce $\Delta F= \pm 1, \Delta M_{F}= \pm 1$ transitions called $\sigma$ transitions which induce the so called Ramsey pulling. Their amplitude is proportional to the tangent of

the angle formed by the static and the microwave fields. The $\pi$ transitions are the $\Delta F= \pm 1, \Delta M_{F}=0$ transitions. Each assymetry in the wings of the neighbouring transitions of the clock transition, ie $F=4, M_{F}=1 \rightarrow F=3, M_{F}=1$ and $F=4, M_{F}=-1 \rightarrow F=3, M_{F}=-1$, will induce a dependance of the piedestal on the static magnetic field. Those transitions induce the so called Rabi pulling. These different couplings (figure 12) induce new atomic paths which will interfere and contribute to the clock signal. This frequency shift cancels when atoms are prepared in the sublevel $\mathrm{M}_{\mathrm{F}}=0$. If the atomic populations of the neighbouring levels are symetric, the Ramsey pulling cancels as well. The weak inhomogeneities of the static magnetic field induce the Majorana transitions $\Delta F=0, \Delta M_{F}= \pm 1$. The curvature of the magnetic field lines at the extremities of the cylindrical magnetic shields are at the origin of the Majorana transitions produced by the existence of Zeeman coherences. This effect is hard to evaluate because it depends on the microwave field as well as the static magnetic field. In order to measure the Ramsey and Majorana pulling, we varied the static magnetic field of few $\mathrm{mG}$ and measure the induced frequency shift of the clock transition (figure 13). A first estimation give a maximal shift of $510^{-16}$ for the Majorana effect and the Ramsey pulling. The frequency shift is quasi-constant at the measurement noise level. The presence of any modulation of this quadratically dependant magnetic field frequency $v_{00}$ would have been a signature of significant Majorana and Ramsey effects.

\section{Conclusion}

This detailed study with the corresponding references give rise to a frequency stability of $410^{-14} \tau^{-1 / 2}$ and an accuracy of $1.410^{-15}$. Those have been the best results ever measured over many years and are still five times better than the ones obtained in the best thermal beam devices. They have been improved later on and are still better than the measurements undergone on the NIST and NRC fountains as the magnetic field control in the Observatoire de Paris device has been fully optimized and is much better than in the other devices. Using the best quartz oscillators would conduct to reach this stability.

The short term frequency stability is limited by the atomic projection noise varying as $\sqrt{\frac{1}{N_{D}}}$ where $\mathrm{N}_{\mathrm{D}}$ is the detected atom number. Measurements have shown that we can obtain a signal to noise ratio of 2000 per 
A laser-cooled atomic probe for low variations of static magnetic fields.

launching supposing $N_{D}$ of the order of $410^{6}$. As pointed out previously, a compromise has to be found between reduced cold collisions and a large SNR. The long term frequency stability is limited by the fluctuations of the atom number which are less than 6\%. This device can be suitable to kill mental diseases and is a good option in neuroimmunology.

\section{Acknowledgments}

The author would like to thank her PhD supervisor A. Clairon and coworkers for constructing the fountain device and its software.

\section{References}

[1]. A. Clairon, S. Ghezali, G. Santarelli, Ph. Laurent, S. N. Lea, M. Bahoura, E. Simon, S. Weyers and K. Szymaniec, Preliminary accuracy evaluation of a cesium fountain frequency standard, 5 th Symposium of frequency Standards and Metrology, Boston, USA. October 1995; A. Clairon, Ph. Laurent, G. Santarelli, S. Ghezali, S. N. Lea and M. Bahoura, IEEE trans. Instrum. Meas., vol. 44, No. 2, April 1995.

[2]. Giorgio Santarelli, PhD thesis, July 1996

[3]. Selma Ghezali, PhD thesis, December 1997.

[4]. G. Santarelli et al, Phys. Rev. Lett. 82, 4619 (1999).

[5]. S. Ghezali et al, EuroPhys. Lett., 36, p. 25, October 1996.

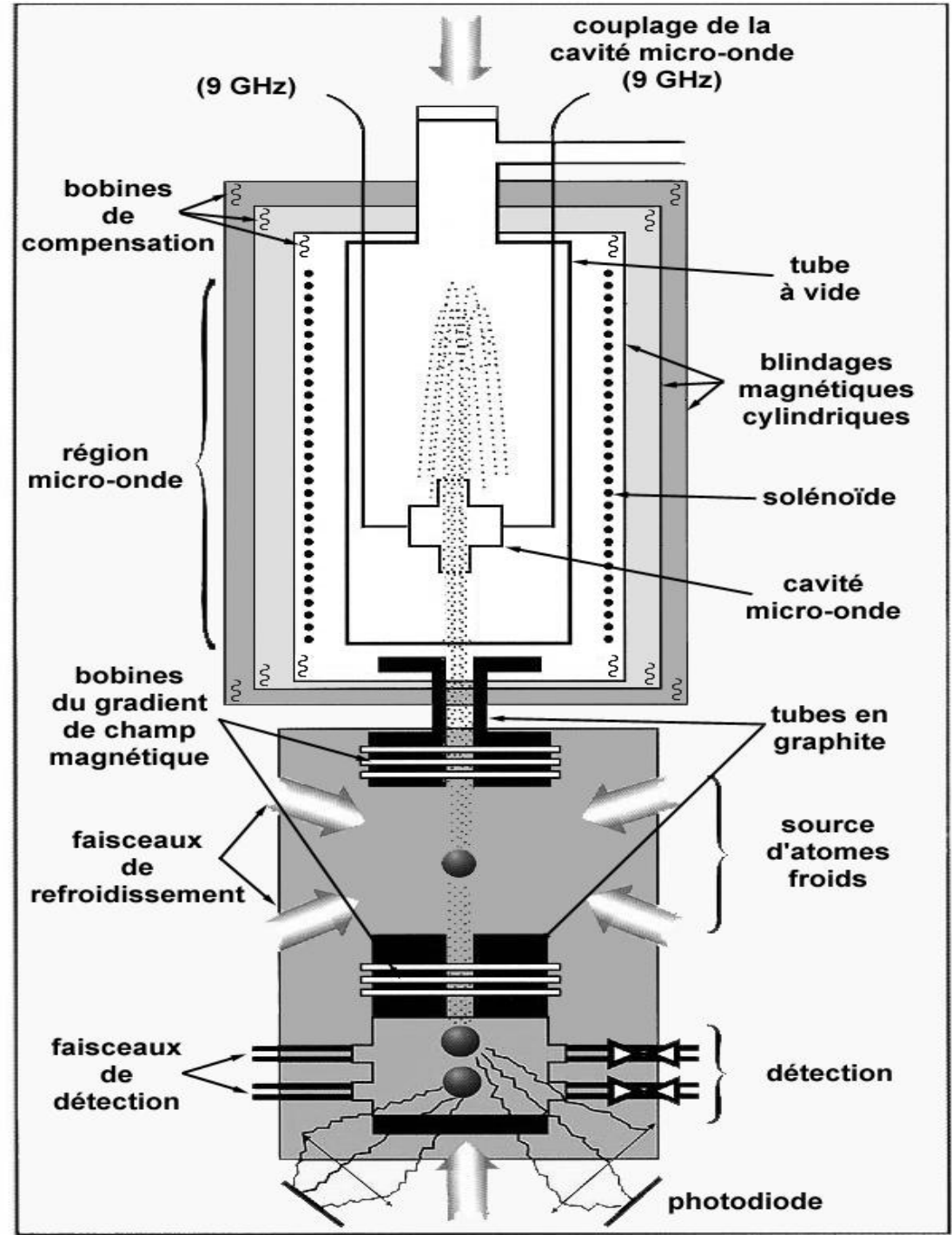

Figure I- 2 : Schéma de la fontaine de Cs

(sans les 8 bobines externes et le quatrième blindage magnétique parallélépipédique)

Figure 1: The fountain device. 


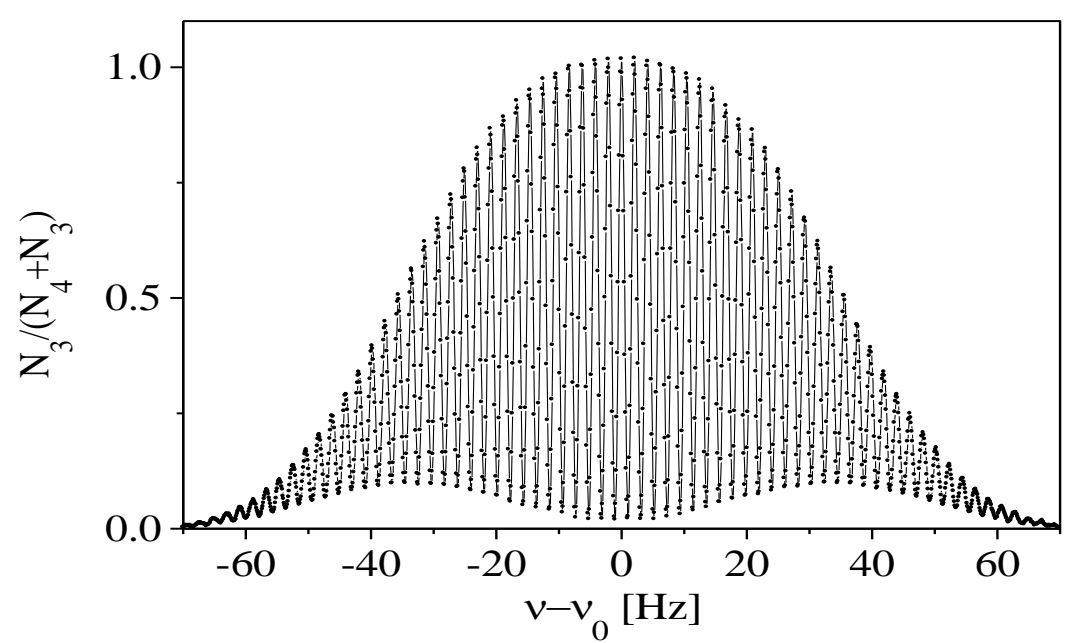

Figure 2: Ramsey fringes.

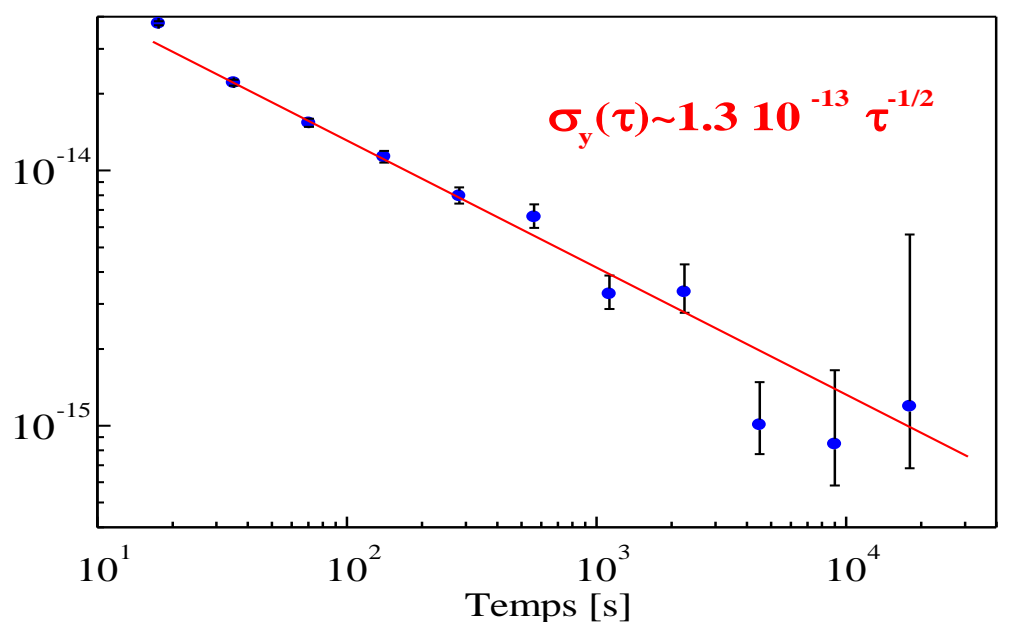

Figure 3: Frequency stability.

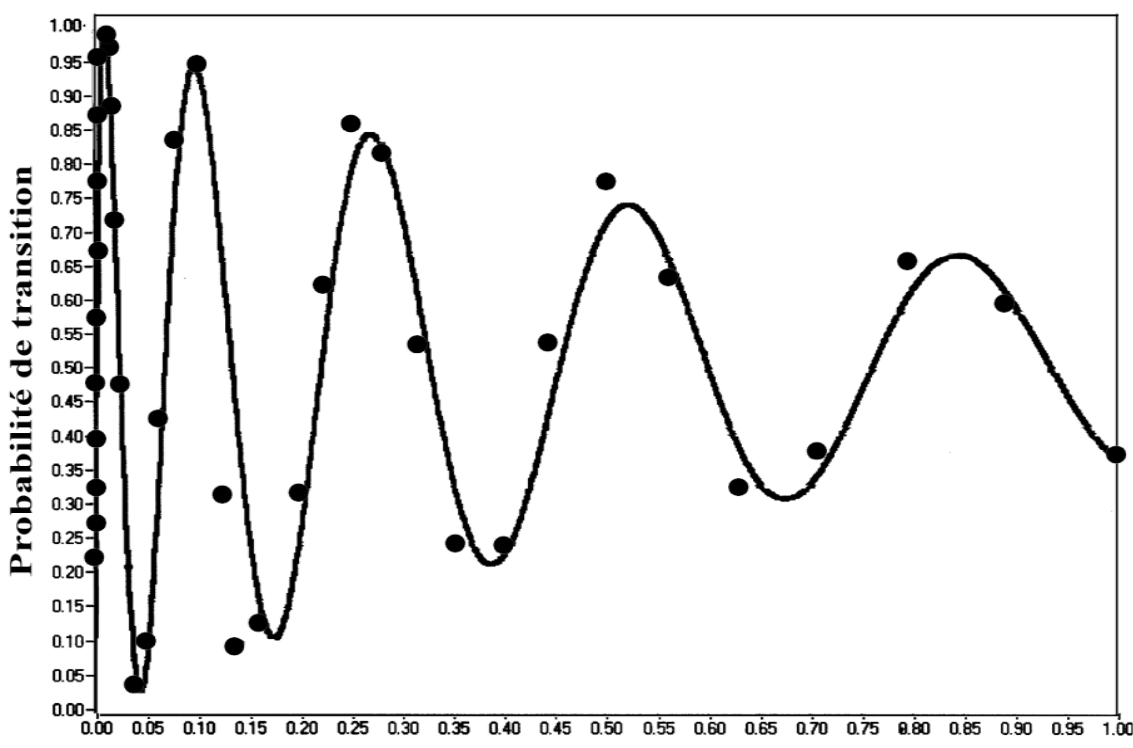

Puissance (u.a)

Figure 4: Rabi oscillations: the transition probability against the microwave power injected in the Ramsey cavity. 


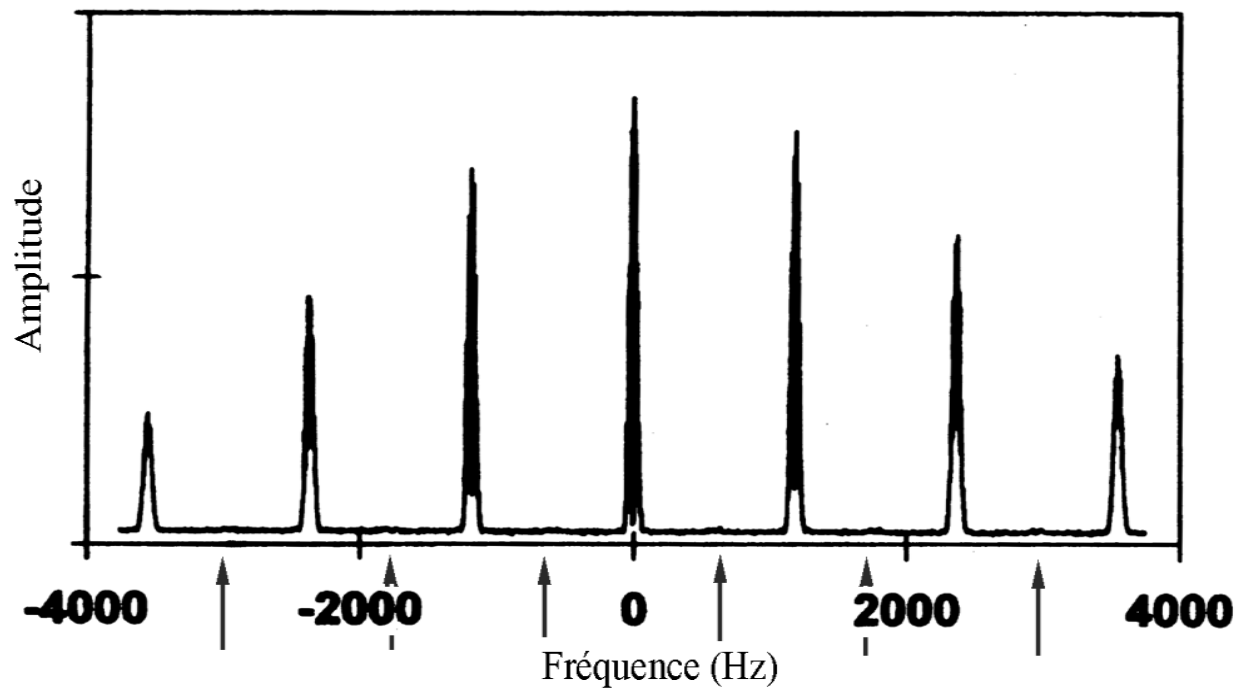

Figure 5: The microwave spectrum.

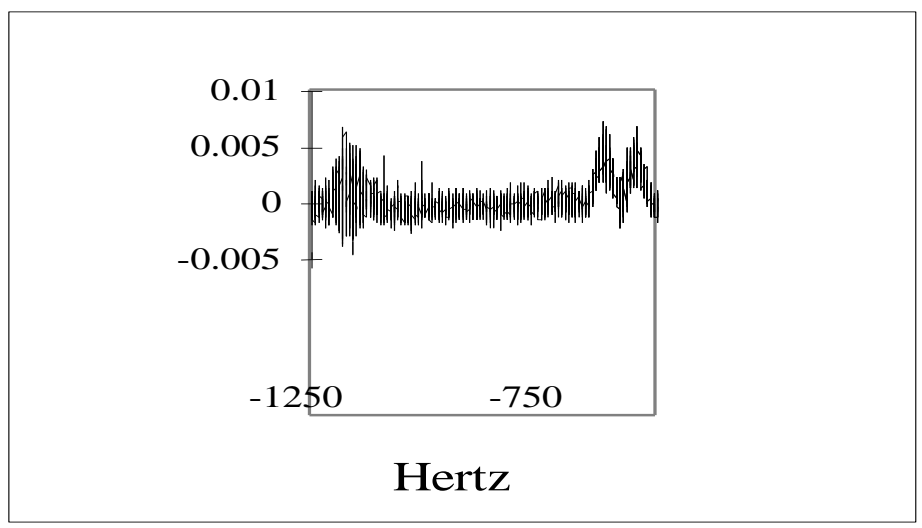

Figure 6: The neighbouring $\Delta \mathrm{M}_{\mathrm{F}}=1$ and $\Delta \mathrm{M}_{\mathrm{F}}=0$ transitions.

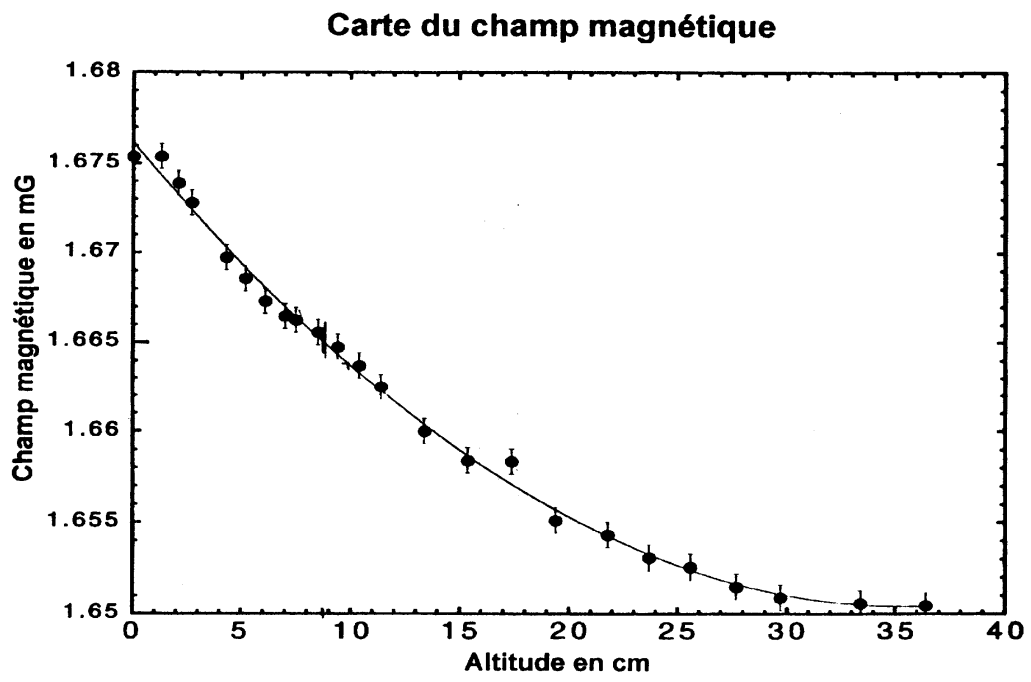

Figure 7: The axial magnetic field map before any correction. 


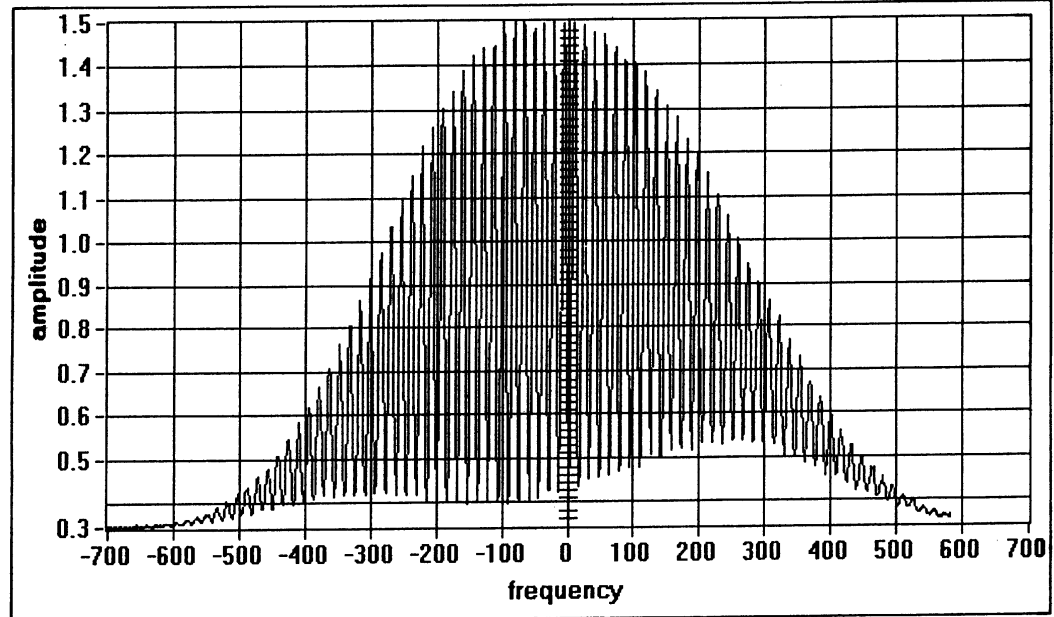

Figure 8: The $F=4, M_{F}=-1 \rightarrow F=3, M_{F}=-1$ transition after the magnetic field correction.

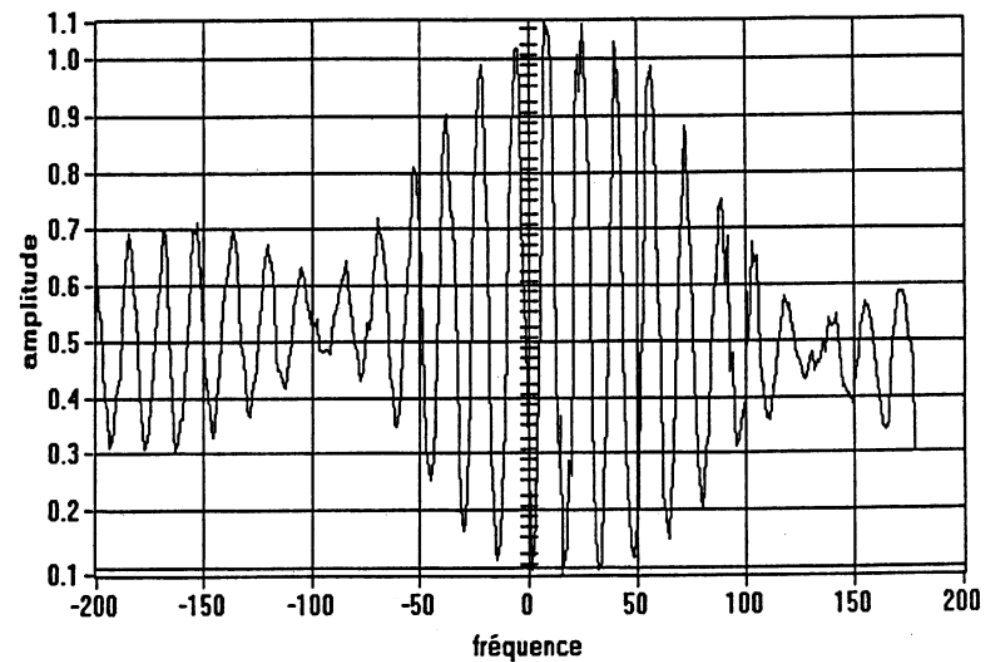

Figure 9: Ramsey fringes for a $\pi$ transition when the velocity distribution is determined by a frequency mixing of the horizontal laser beams.

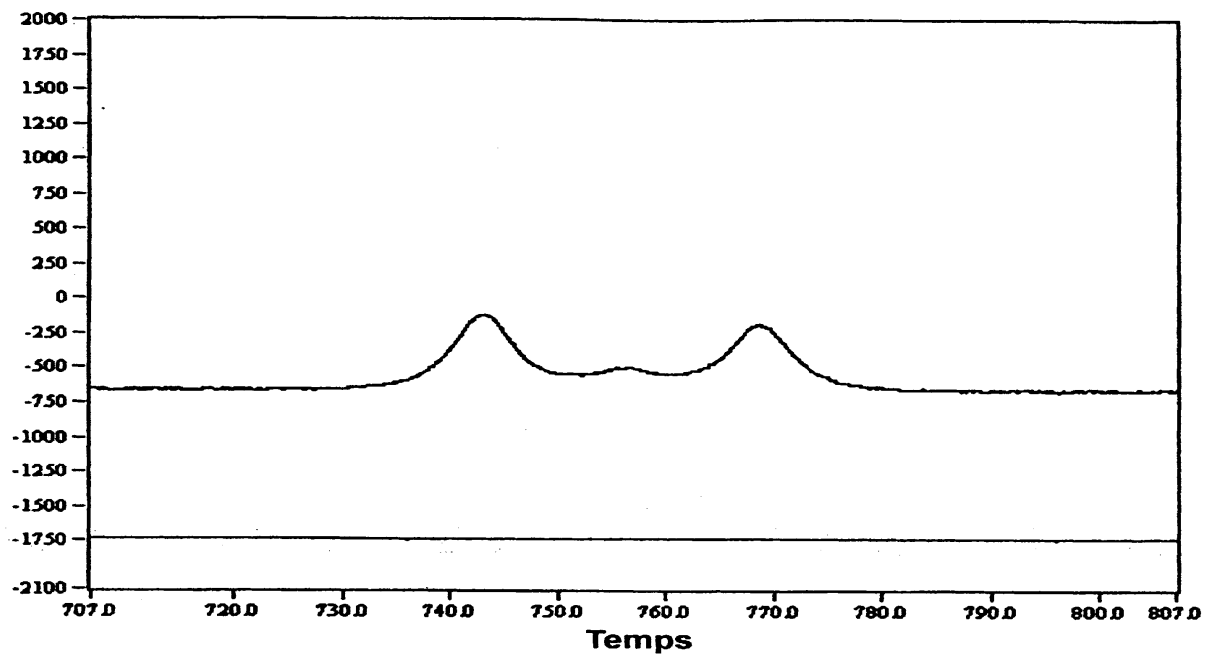

Figure 10: A time of flight with 3 pics corresponding to a frequency difference between the horizontal laser beams during the acceleration phase of the order of $+60 \mathrm{kHz}$. 


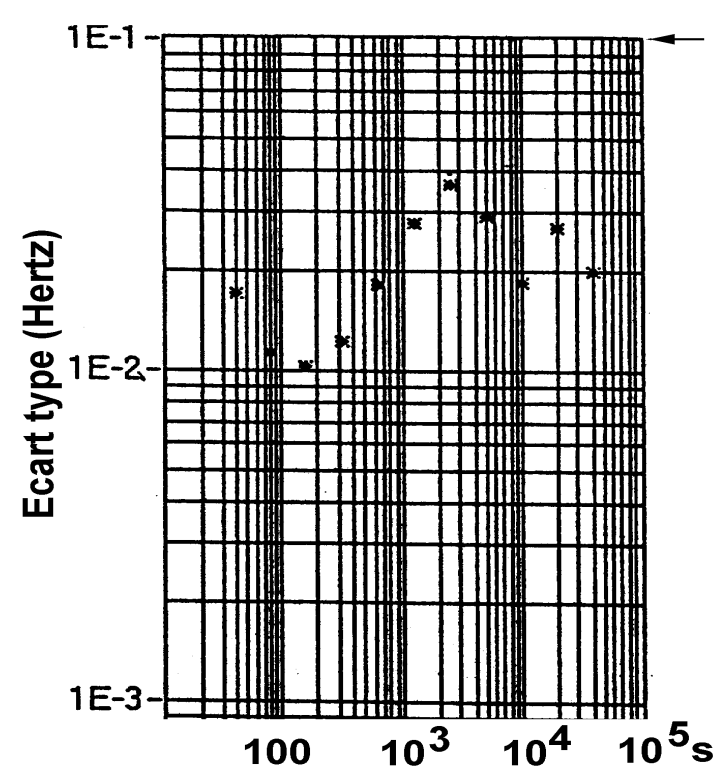

Figure 11: The short term stability of the static magnetic field.

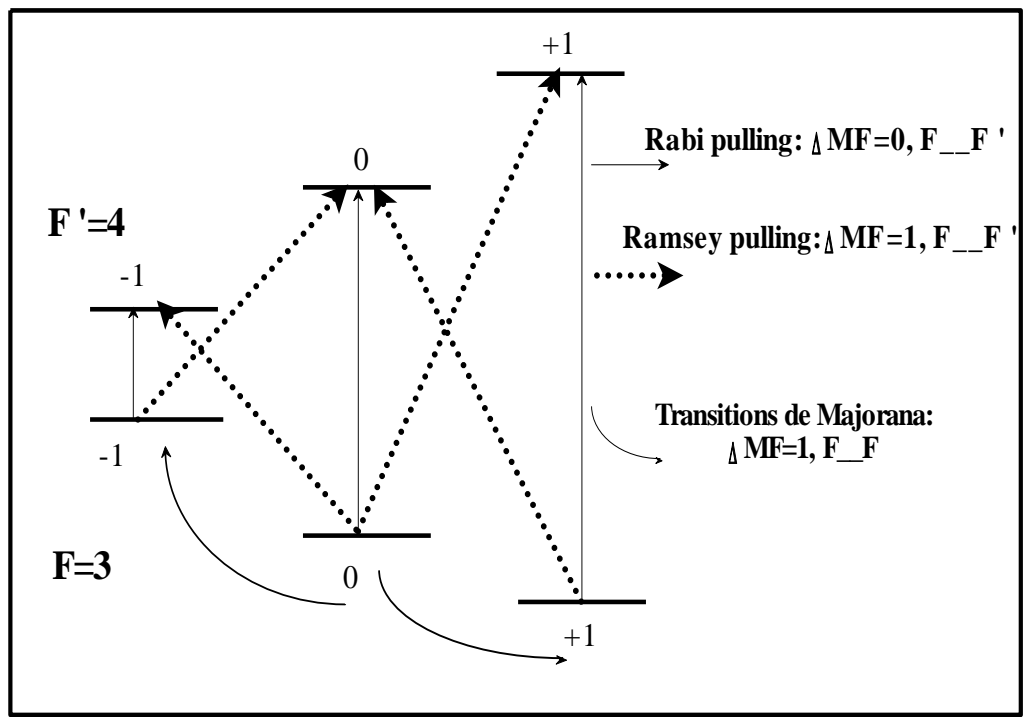

Figure 12: The atomic structure with the different possible couplings $\sigma$ and $\pi$.

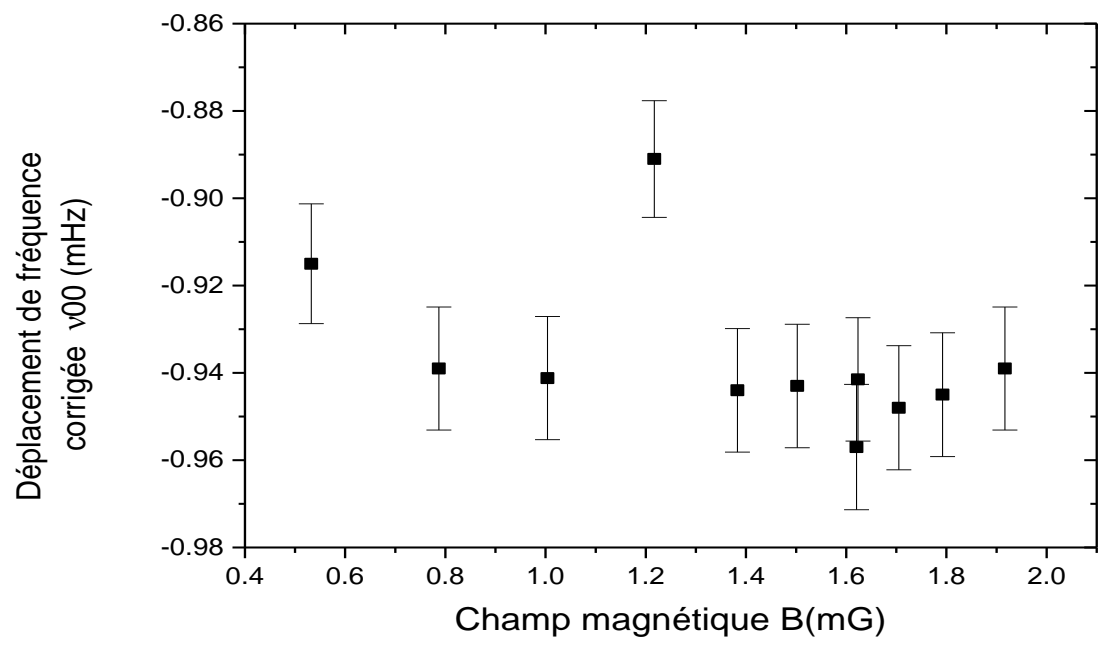

Figure 13: The corrected Zeeman frequency shift of the clock transition. 